\title{
Improved test setup for MEMS mechanical strength investigations and fabrication process qualification
}

\author{
T. Bandi ${ }^{* a b}$, X. Maeder ${ }^{a}$, A. Dommann ${ }^{c}$, H. Shea ${ }^{\text {b }}$ and A. Neels ${ }^{a}$ \\ ${ }^{a}$ CSEM, Microsystems Technology Division, Jaquet Droz 1, 2002 Neuchâtel, Switzerland; \\ ${ }^{b}$ EPFL, Microsystems for Space Technologies Laboratory, Rue de la Maladière 71b, 2002 Neuchâtel, Switzerland; \\ ${ }^{c}$ EMPA, Department Materials meet Life, Überlandstrasse 129, CH-8600 Dübendorf
}

\begin{abstract}
The mechanical stability of silicon MEMS dies is strongly influenced by the microfabrication processes, especially grinding, dicing and etching, which leave characteristic damage (defects, cracks, dislocations...) in the substrate material. Specially designed mechanical tests are used to assess the resistance of micro-structures to monotonic and cyclic loading. We report on the development progress of a micromechanical test bench for reliability assessment of microstructures in 2-, 3- and 4-point bending configurations. Strain distributions and defects in micron-sized silicon devices can be investigated by in-situ testing in combination with high-resolution x-ray diffraction measurements for experimentally assessing the strain distribution.
\end{abstract}

Keywords: Micromechanical test, Bending test, Reliability, Silicon, Micromechanics, Micro-electromechanical systems, Yield strength.

\section{INTRODUCTION}

Microfabrication of single crystal silicon allows using its unique combination of high material quality, low density, high Young's Modulus, resistance to fatigue and batch processing with low fabrication tolerances in a wide range of geometries and electro-mechanical applications [1]. However, the quality of the crystal in a finished MEMS device is influenced by the fabrication and packaging processes, many of which introduce defects that deteriorate the mechanical stability of the material. Due to the brittle nature of silicon these defects can lead to catastrophic failure during assembly, packaging and operation.

Die separation methods such as deep-reactive ion etching (DRIE), mechanical blade dicing and laser dicing induce characteristic damage near the cutting lines. Grinding and polishing used for wafer thinning leave scratches and marks on the backside of the wafer. More specifically, blade dicing induces chipping and cracking near the cutting line and laser dicing can lead to cracks and zones with high defect densities near the cut surface [2]. Deep-reactive ion etching is an iterative process in which isotropic etching is alternated with a passivation of the sidewalls, giving rise to near-vertical but scalloped and rough trenches in the silicon by means of which the structures are defined [3].

By characterization of the failure modes and failure distributions, micromechanical tests support the qualification and optimization of fabrication and die separation processes, allowing to improve the yield and reliability of systems. Because it is prohibitively complex and time-consuming to test all possible geometries used in MEMS devices it is preferable to use test specimens (e.g. straight beams) to predict the failure distribution of structures with arbitrary shapes [4]-[7]. Moreover, this allows benchmarking new technologies and processes against existing fabrication methods. Nevertheless, the mechanical test setup should allow reproducing the strain conditions and sample properties as close as possible to MEMS operation due to the following reasons. First, the distribution of damages varies with the depth of the

*Tobias.Bandi@csem.ch; phone +41 32720 5207; fax +41 32720 5700; csem.ch

Reliability, Packaging, Testing, and Characterization of MOEMS/MEMS, Nanodevices, and Nanomaterials XIII, edited by Herbert R. Shea, Rajeshuni Ramesham, Proc. of SPIE Vol. 8975, 897509

(c) 2014 SPIE · CCC code: 0277-786X/14/\$18 · doi: 10.1117/12.2044212 
processed structure. Examples are two-step blade dicing [2] and the variation of the damage with increasing depth of plasma etching [4], which influences the overall strength of structures.Secondly, cracks initiated at the strained surface and at the strained edges both contribute to the failure distribution of silicon dies [8]. Surface and edge flaws were observed to be the main source of chip fracture in Flip-chip assemblies due to stresses built up by soldering and thermal cycling [9],[10]. Therefore it is important to reproduce the thickness and the loaded-surface-to-edge ratio (i.e. the width of the sample when the thickness is given) as well as the strain directionality as close as possible when simulating stresses occurring in real devices by mechanical testing.

The applications targeted here are specimens with sub-mm width and thicknesses of 50-500 $\mu \mathrm{m}$. For instance microfabricated structures used in mechanical watches or macro-MEMS (3D sensors and actuators made from 2D building blocks fabricated from wafers [12]) can experience very high levels of mechanical strain during assembly and operation. In addition, the constant device miniaturization in advanced technologies leads to dies size which are smaller than $1 \mathrm{~mm}^{2}$, for instance in supervisory and RFID circuits.

Numerous test setups for evaluating the mechanical reliability of wafers and chips have been developed and reviewed in [13]-[15]. For testing the effect of grinding and polishing, rotationally symmetric bending tests such as the ball-ring test or the ring-ring tests are suitable [16]. The edges are not loaded in these test geometries and hence they are sensitive to surface damages. Specialized test geometries such as the twist-bending test or the ball-on-edge test are sensitive to (wafer) edge flaws but the distribution of the stress along the edges is very sensitive to the position of the supports and the stress level itself [13]. Miniaturization of the test setup therefore is challenging. The $n$-point bending tests $(n$ equals to 2,3 or 4) allow testing for edge and surface flaws simultaneously.

Recently an instrument for displacement controlled two-point bending which allows testing specimens with dimensions similar to typical microfabricated silicon structures has been presented [17]. The specimen was a long, slender beam with a cross-section of $50 \times 50 \mu \mathrm{m}^{2}$ and a length of $5 \mathrm{~cm}$. During the test it was bent in a U-shape and kept between two parallel chucks by its own elasticity. The maximum stress occurred at the apex of the non-linearly bent beam while at the contact points the beam was almost straight. An advantage of this test geometry is therefore that the contact points of the chucks are far away from the areas with maximum stress which prevents fracture due to additional clamping and contact stresses. During the test the two chucks were approached continuously and the elastic force exerted by the specimen on the chucks was monitored until fracture occurred.

As the specimen is fixed by its own elasticity in this test it must be able to support a minimum stress at the beginning of the test. For a specimen of $50 \mu \mathrm{m}$ thickness is was approximately $1 \mathrm{GPa}$ which is beyond the strengths achievable by standard wafer dicing processes.

This study will focus on the further development of this instrument to extend the range of testable yield strengths by implementation of 3-point and 4-point bending. Three- and four-point bending test methods are commonly made in the linear regime where the bending of the specimens is negligible. However, due to the small width and thickness of devices, which can be below $100 \mu \mathrm{m}$, the non-linearity of the bending must be considered. Then the experimental setup for the semi-static characterization of the test beams is described before the results are presented and discussed.

\section{BENDING TEST THEORY AND SIMULATIONS}

As mentioned above it is important to reproduce fabrication processes and specimen geometries of real devices as close as possible in when evaluating the failure distribution of test specimens such as beams. The length of the beams is less crucial because it can be accounted for by analysis of the Weibull failure distribution. The probability of rupture $S$ in the 2-paramter Weibull distribution is given by [11]

$$
S=1-e^{-\int_{A} k\left(\frac{\sigma}{\sigma_{0}}\right)^{m} d A}
$$

In (1) the exponent is integrated over the whole stressed surface. This is justified by the assumption that defects at the surface cause fracture; otherwise the integration may run over the stressed volume. The parameters $\sigma, \sigma_{0}, m$ and $k$ denote the stress at a given position on the surface of the specimen, the characteristic yield strength, the shape parameter of the Weibull distribution and a proportionality factor which equals unity. The parameters $\sigma_{0}$ and $m$ are the characteristic parameters of the failure distribution and depend on the material properties and defect distribution. If the sample length 
is varied this will increase the total loaded area but not change the defect distribution, and hence $\sigma_{0}$ and $m$, because they do not vary along the length of the specimen if the cross-section is constant (as is the case for rectangular cuboid dies).

In [18] the non-linearity of three-point bending and four-point bending was analyzed by a spring model with rigid bodies and by finite element analysis (FEA). A reasonable correspondence between the experimental three-point bending and the FEA model was found, but the case of four-point bending has not been experimentally validated. Here the non-linear bending of the specimen was numerically simulated by considering the large deflection of a slender Euler-Bernoulli beam. In the bent beam the outer exposed side is under tensile strain, the inner side is under compression and the neutral centerline is stress-free. The stress increases linearly with the distance from the centerline, and hence thicker specimens are under higher stresses at a given beam curvature than thin specimens. The coordinate system is chosen such that the unconstrained beam is parallel to the $\mathrm{x}$-axis and during the test it is deflected in the $\mathrm{y}$-direction. In all cases the positions of the pins along the $x$-axis is equidistant. The bending of the beams in the three-point bending is given by the following second order differential equation:

$$
\frac{M}{E I}=\frac{1}{E I} F\left(x(\cos (\theta)+\mu \sin (\theta))+y\left(\sin (\theta)-\mu \cos (\theta)=\frac{\frac{\partial^{2} y}{\partial x^{2}}}{\left(1+\left(\frac{\partial y}{\partial x}\right)^{2}\right)^{3 / 2}}\right.\right.
$$

The parameters $E, I, F, x, y, \theta$ and $\mu$ are the Young's modulus, second moment of inertia, the force acting on the outer pins normal to the beam, the $x$ and $y$ coordinate of a point on the beam centerline, the contact angle of the beam with the outer pins and the static friction coefficient between the pins and the beam, respectively. Equation (2) was numerical solved using a Runge-Kutta approach similar to the technique proposed in [19] for four-point bending: The integration was made starting from the outer pins towards the center of the beam. It was limited to one half or the beam profile due to the symmetry of the profile. For a given contact angle $\theta$ an initial guess was made for $F$ and then equation (2) was solved stepwise using the Runge-Kutta algorithm [20]. At the apex of the beam profile its gradient needed to vanish. If this condition was not fulfilled the force $F$ was adjusted and the process iterated until convergence was achieved. In the four-point bending a similar expression for the bending moment $M$ applies but the two zones between the outer and the inner pins and between the inner pins and the center need to be treated separately [19].

The size of the pins exerting the bending force on the specimens was non-negligible in comparison to the specimen size and because large bending angles were encountered during the tests, the model was extended to account for the effect of the pins on the profile of the bent beam.
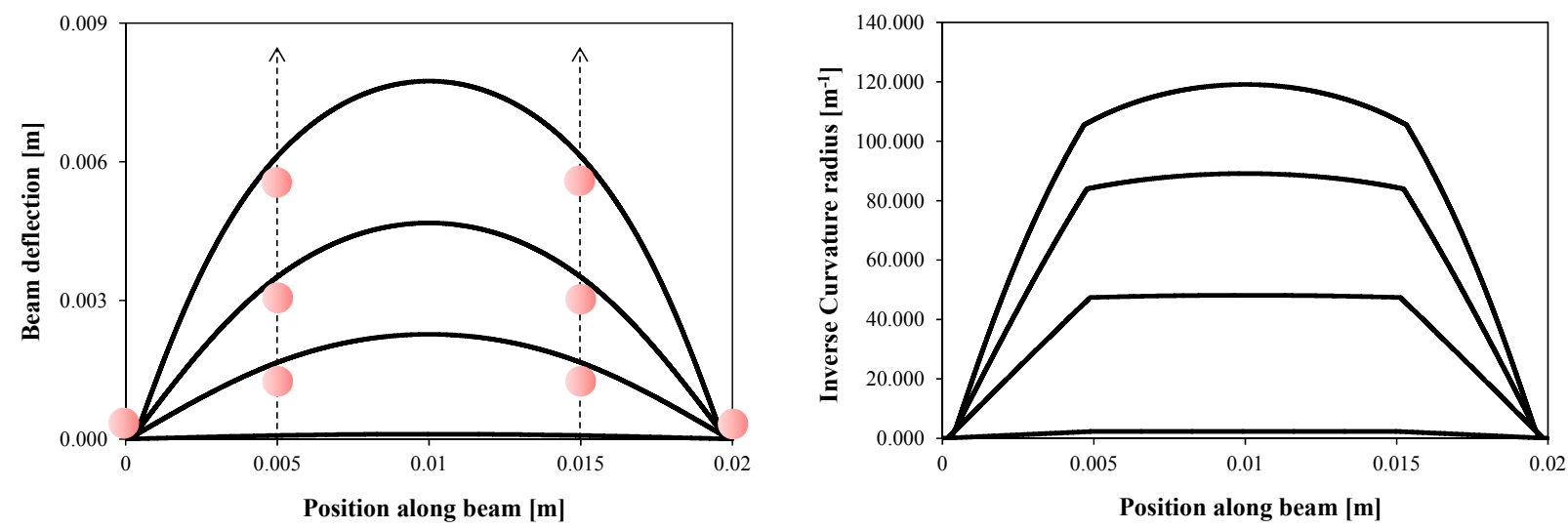

Figure 1: (Left) Profile of a non-linearly bent Euler-Bernoulli beam for various deflection states. The round dots show the position of the pins. (Right) The inverse curvature radius along the beams. 


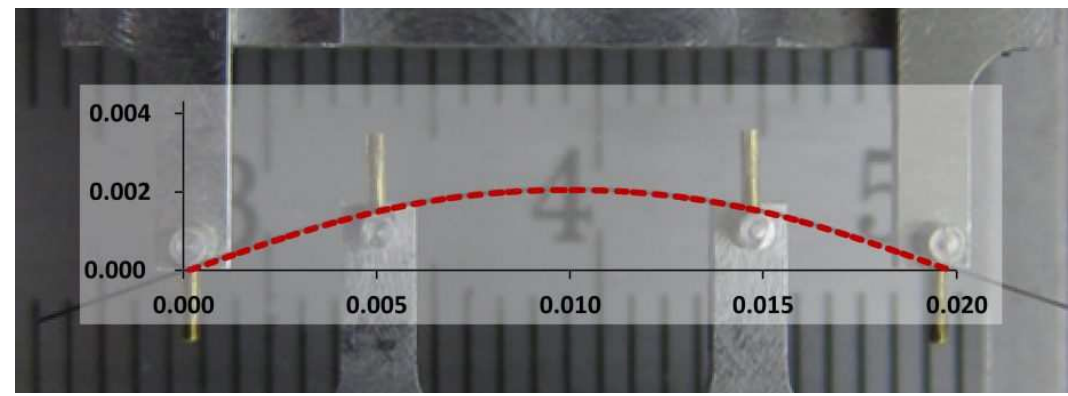

Figure 2: Superposition of a bent beam and the theoretical beam profile according to equation (2). The force sensor is attached to the inner pins while the outer pins are displaced by the stepping motor. The units of the $x$ and $y$ axis is meters.

This was simply achieved by calculating the contact point on the pins as a function of the contact angle. In Figure 1 left, the profile of the beam for various deflections in the case of four-point bending is shown. The round points show the position of the pins which enforce the bending of the beam. The right graph in Figure 1 shows the inverse curvature radius along the bent beam.

The profile and curvature of the beam depend on the static friction coefficient $\mu$ between the beam and the pins. Therefore the 3-point and 4-point bending tests also yield information on this parameter. Based on the simulations the experimentally accessible force of the beam on the pins parallel to the y-axis was calculated.

Figure 2 shows a comparison of the profile of a bent beam with the profile obtained by solving equation (2). The calculated bending line (red) was uniformly scaled and superposed over the experimental image and an excellent agreement was found.

\section{EXPERIMENTAL SETUP}

As mentioned above the experimental setup was based on the instrument presented in [17]. The two chucks which enforced the bending of the beam were mounted on a stepping motor and a force sensor, respectively. The motor was moved at a speed of $10 \mu \mathrm{m} / \mathrm{s}$. The pins were made from Aluminum and had a radius of $0.5 \mathrm{~mm}$ to avoid sharp contacts. To qualify the experimental setup single-crystal silicon beams fabricated by deep-reactive ion etching with a crosssection of $50 \times 50 \mu \mathrm{m}^{2}$ and $50 \mathrm{~mm}$ length were used. The same specimens were used in 2-point bending in [17], so the dimensions, surface state and failure distribution were well characterized. The characteristic yield strength and the shape parameter of the 2-parameter Weibull distribution were $4 \mathrm{GPa}$ and 2.62, respectively. The etched sidewalls were oriented perpendicular to the bending direction (i.e. out of the image plane in Figure 2).

During the experiment the displacement of the stepping motor was recorded together with the force $P$ acting on the pins normal to the direction of motion of the pins (y-axis in Figure 1). For comparing the experiments of specimens with different sizes the force was normalized with regards to the distance $a$ between the outer pins, the elasticity of the beams and their second moment of inertia $\alpha=2 P a^{2} / E I$.

\section{RESULTS AND DISCUSSION}

Figure 3 shows the experimental results obtained in three-point bending (Figure 3, left) and four-point bending (Figure 3, right). The maximum strain to which the specimens were exposed in the two tests was around $1 \mathrm{GPa}$ in both tests. Therefore the specimens did not break even at the highest deflections. This allowed measuring the bending parameters of the specimens in the full experimentally accessible range. In the linear-bending regime where the experimental data overlaps with the dotted line (linear theory) the repeatability of the data was best. At higher deflections the variations of the data increased, which was attributed to a stepped slipping motion of the beam along the pins. 

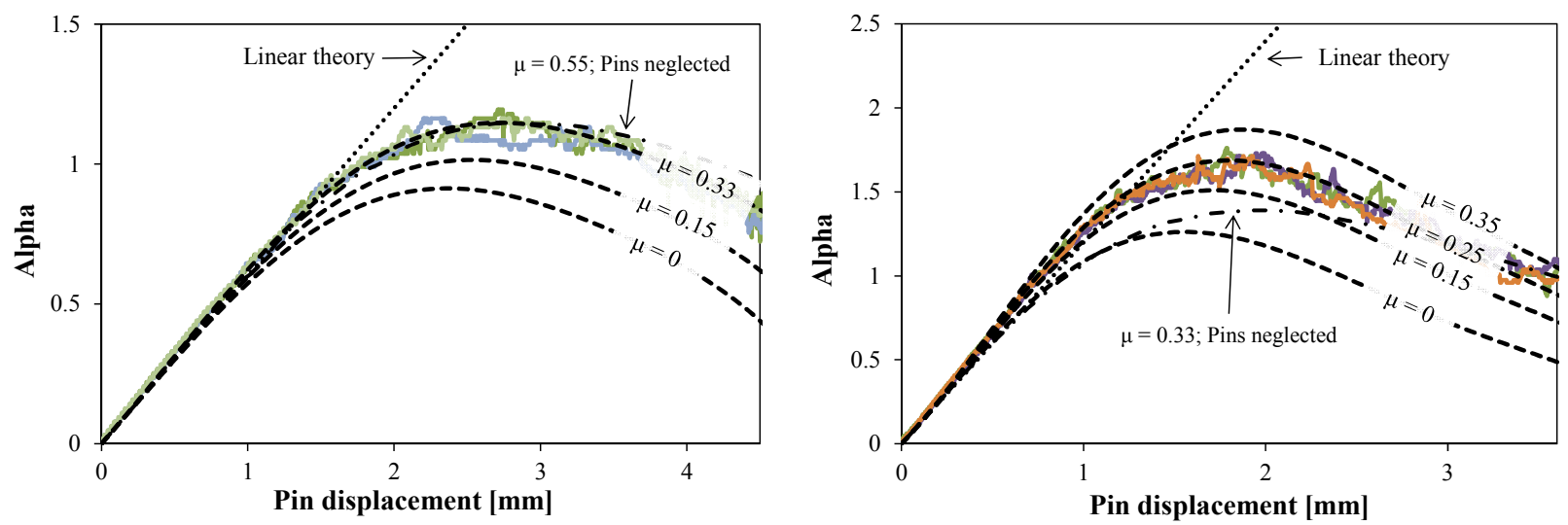

Figure 3: Force versus pin-displacement for the three-point bending (Left) and four-point bending (right). The distance between the outer pins was $10 \mathrm{~mm}$ in both cases. Three measurements with three different samples are shown in both graphs. The dotted line represents the linear theory, the dashed line shows the results of the non-linear bending theory, and the dash-dotted line is the nonlinear model without considering the pin geometry.

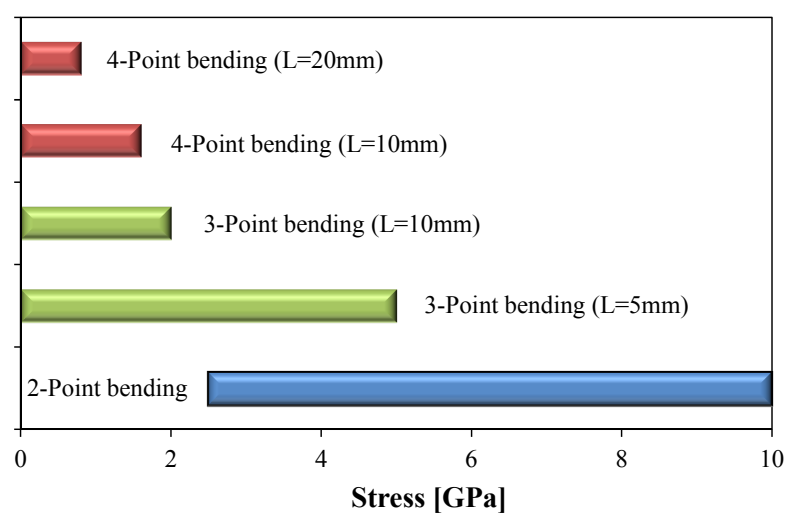

Figure 4: Comparison of the experimentally accessible stresses in a $100 \mu \mathrm{m}$ thick specimen. The values of $L$ denote the distance between the outer pins.

The experimental data showed a very good agreement with the model with static fiction coefficients of 0.25 and 0.33 for the four-point bending and the three-point bending, respectively. The variation between the two values was presumably due to variations of the surface finish of the aluminum pins.

For comparison the theoretical curves for various values of the friction coefficient (dashed lines). The dash-dotted line shows the simulated result under neglect of the pins (radius equal to zero). The significant disagreement between this model and the experimental data shows the importance of incorporating the pins in the model.

The results obtained show that the instrumental setup for three-point bending and four-point bending provides valuable insights on the mechanical properties and failure distribution of microfabricated specimens under bending loads. By adapting the size of the chucks different stress ranges can be accessed. To exemplify this, Figure 4 shows the experimentally accessible stress ranges for a sample with a thickness of $100 \mu \mathrm{m}$. By selecting the appropriate test geometry the mechanical stability of specimens with widely varying yield strengths can be evaluated.

\section{CONCLUSIONS}

The development progress of a micromechanical test bench for the reliability assessment of microstructures was reported. The instrument induces a displacement-controlled deformation in 4-point, 3-point and 2-point bending. The mechanical test setup allows reproducing the strain conditions and sample properties such as surface-to-volume ratio and loaded-surface-to-edge radio as well as the fabrication processes as close as possible. In addition, tensile testing 
geometries and custom-made chucks for testing of specific specimen geometries are available [17]. The instrumental test setup allows quantifying the failure distribution in test structures and functional devices with yield strengths in the full range between 0 and $10 \mathrm{GPa}$. In addition, in-situ testing in combination with high-resolution x-ray diffraction measurements (HRXRD) can be used to investigate strain distributions and defects in crystalline specimens [17].

By quantifying the yield strength distributions these methods allow optimizing fabrication and die separation processes. The safety margins of structure geometries and of dicing lines may be reduced which can lead to significantly higher number of devices per wafer, most importantly for small dies with surfaces of below one square-millimeter. Further results on the qualification of laser processes for chip separation using HRXRD combined with micromechanical testing shall be reported on in detail elsewhere.

\section{ACKNOWLEDGMENTS}

This work was supported in part by the Networking/Partnering Initiative of the European Space Agency under Contract No. 21872. We thank Laurent Marchand and Nicolas Saillen from the Materials and Components Division of the European Space Agency for the continued project support and collaboration.

\section{REFERENCES}

[1] Petersenn K. E., "Silicon as a mechanical material," Proc. IEEE 70(5), 420-457 (1982).

[2] Lei W-S., Kumar A. and Yalamanchili R., "Die singulation technologies for advanced packaging: A critical review," J. Vac. Sci. Technol. B 30, 040801 (2012).

[3] Gaither M. S., Gates R. S., Kirkpatrick R., Cook R. F. and DelRio, F. W., "Etching process effects on surface structure, fracture strength and reliability of single-crystal silicon theta-like specimens," J. Microelectromech. Syst. 22(3), 589-602 (2013).

[4] Matthewson M. J., and Nelson G. J., "A novel four-point bend test for strength measurement of optical fibers and thin beams - Part II: Statistical Analysis," J Lightwave Technol. 14(4), 564-571 (1996).

[5] Huy V. L., Kamiya S., Nagayoshi K., Izumi H., Gaspar J. and Paul O., "A prediction scheme of the static fracture strength of MEMS structures based on the characterization of damage distribution on a processed surface," J. Micromech. Microeng. 23, 045008 (2013).

[6] Steiert M. and Wilde J., "New probabilistic reliability model describing the risk of chip fracture in chip-onboard technology," 4th Electronic System-Integration Technology Conference (ESTC) 17-20 Sept. 2012, 1-6, (2012).

[7] Boyce B. L., Ballarini R. and Chasiotis I., "An argument of proof testing brittle microsystems in high-reliability applications," J. Micromech. Microeng. 18, 117001 (2008).

[8] Chae S.-H., Zhao J.-H., Edwards D. R. and Ho P. S., "Effect of dicing technique on the fracture strength of Si dies with emphasis on multimodal failure distribution," IEEE T. Device Mat. Re. 10(1) 149-156, (2010).

[9] Ranjan M., Gopalakrishnan L., Srihari K., and Woychik C., "Die cracking in flip chip assemblies," Proc. 48th IEEE Electronic Components \& Technology Conference, 729-733 (1998).

[10] Tsai M-Y., Hsu C. H. J. and Wang C. T. O., "Investigation of thermomechanical behavior of flip chip BVA packages during manufacturing process and thermal cycling," IEEE T. Compon. Pack. T. 27(3), 568-576 (2004).

[11] Weibull W., "A statistical theory of the strength of materials," Proc. Roy. Swedish Inst. for Eng. Res. 151, 5 (1939).

[12] Henein S., Barrot F., Dommann A., Droz S., Lisowski L., Mayor J.-M., Overstolz T., Schwab P., Walter P. and Verjus C., "Silicon flexure-based micro-balance for batch weighing processes," CSEM Scientific and Technical report (2008), <http://www.csem.ch/docs/Show.aspx/9407/docname/CSEM-STR08-Page\%2089.pdf> (30. December 2013).

[13] Brückner J., Auerswald E., Dudek R., Rzepka S. and Michel B., "Review of experimental strength testing methods for silicon dies," Proceedings of MicroCar 2011, March 1st Leipzig (D), 2011.

[14] Yeung B. and Lee T-Y. T., "An overview of experimental methodologies and their applications for die strength measurement," IEEE T. Compon. Pack. T. 26(2) 423-428, (2003). 
[15] Tsai M-Y. and Lin C. S., "Testing and evaluation of silicon die strengths," IEEE T. Electron. Pa. M. 30(2), 106114 (2007).

[16] Behnken H., Ape M. and Franke D., "Simulation of mechanical stress during bending tests for crystalline wafers," Proceedings of 3rd World Conference on Photovoltaic Energy Conversion 2, 1308-1311 (2003).

[17] Schifferle A., "Combined experimental methods for the mechanical characterization of materials", PhD Thesis, ETH Zürich (2011).

[18] Schoenfelder S., Ebert M., Landesberger C., Bock K. and Bagdahn J., "Investigations of the influence of dicing techniques on the strength properties of thin silicon," Microelectronics Reliability 47, 168-178 (2007).

[19] Nelson G. J., Matthewson M. J. and Lin, B., "A novel four-point bend test for strength measurement of optical fibers and thin beams - Part I: Bending Analysis," J Lightwave Technol. 14(4), 555-563 (1996).

[20] Bronstein I. N., Semendjajew K. A., Musiol G. and Mühlig H., [Taschenbuch der Mathematik], Harri Deutsch, Verlag Harri Deutsch, Thun, (2001), in german. 